\title{
Optimization of Control System Structure and Practice in Engineering Education
}

\author{
Jian Xue ${ }^{1, a}$, Ping $\mathrm{Xin}^{1, \mathrm{~b} *}$ and Yanming Cheng ${ }^{1, c}$ \\ ${ }^{1}$ College of Electrical and information engineering, BeiHua University, Jilin132021, China;

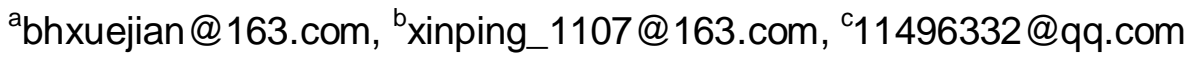 \\ *The corresponding author
}

\begin{abstract}
In the field of control, with the intensification of social competition and the influence of the theory and engineering series of engineering disciplines, the study of control courses has become one of the basic theories and practical bases that modern engineering high-level talents should have, The ultimate goal of the course should be to return to engineering practice and guide the engineering practice. In the past, the teaching and practice mode is simple, the teaching method and the experimental conditions limit the students' learning subjective initiative and the improvement of learning interest. Therefore, it is imperative to optimize the curriculum system, innovation teaching method and practice teaching reform. This paper mainly elaborates the optimization of curriculum structure and practice, adopts modern teaching methods, innovative teaching mode and new learning environment, and provides students with practical learning experience close to engineering.
\end{abstract}

Keywords: Control curriculum; Theory system; Practice system; Course reform; Engineering education;

\section{面向工程教育的控制类课程体系结构及实践环节}

\section{的优化}

\author{
薛健 $^{1}$, 辛平 ${ }^{1}$, 程艳明 ${ }^{1}$ \\ (1. 北华大学电气信息工程学院, 吉林省 吉林市 132021)
}

摘要: 在控制领域中, 随着社会竞争的加剧和工程专业的控制理论与工程系列课程影响力的提高, 控制类课程的学习已 经成为现代工程高层次人才所应具备的基本理论和实践基础之一, 课程的最终目的应该是回归工程实践, 为工程实践作指导。 以往的教学和实践模式单一, 教学方法和实验条件限制了学生学习主观能动性的发挥和学习兴趣的提高, 因此优化课程体系、 创新教学方法和实践教学改革势在必行。本文主要阐述对课程体系结构及实践环节的优化, 采用现代教学手段、创新的教学 模式和新的学习环境, 为学生提供接近工程实际的学习经验。

关键词;控制类课程；理论体系；实践体系；课程改革；工程教育 引言

CDIO 工程教育模式是近年来国际工程教育改革的新成果, 在世界各地多所大学进行了实施并取得很好 的成效。CDI0, 即构思 (conceive)、设计 (design)、实施 (implement)、运作 (operate), 是以现代工业产 品从构思、研发到运行乃至终结废弃的全生命周期为蓝本的、旨在培养学生的工程能力的一种方法。CDIO 在中国的普及和实践, 也为高质量实现 “电气信息类息专业控制理论平台的建设” 打下了良好的基础, 起 到了积极的推进作用。在我校建设电气信息类专业控制理论平台中, 如何适应 CDIO 工程教育模式目标, 使学生在实际动手能力、独立创新能力、团队合作能力方面有所提高, 具有研究意义和现实意义。控制类 
课程是工程类学生的重要基础课, 也是理论性和工程实践性并重的专业基础课。在控制领域中, 只有将课 程理论与实践并重, 才能培养出工程教育所要求的具有工程意识、实践能力和团队合作的综合素质人才。

\section{1 课程体系结构的优化}

针对目前课程体系结构在教学中所体现的知识结构不合理、理论教学与实践教学的不平衡、教学方法 的落后和学生创新实践能力的不足等问题, 我们对课程体系结构进行优化, 真正形成 “教一学一练一研一 用” 五点一线的知识体系势在必行, 采用现代教学手段、创新的教学模式和新的学习环境的引入也会为学 生提供接近工程实际的学习经验。

\section{1 优化教学内容, 精炼教学核心知识点}

教学内容优化是教学改革的核心点, 同时也决定着理论教学与实践能否衔接成功。传统的理论教学存 在很多弊端, 如: 运用数学的力度大, 抽象性强, 学生只是机械地记住公式, 面对具体工程问题却不知如 何下手, 在教学内容的改革中, 主要精炼知识结构, 贴合工程实际。

教学内容优化集中在以下几个方面:

(1) “三基” 要强化:

即基本概念、基本理论、基本方法要扎实，牢固，才能为实践服好务;

(2) “工程实例” 要引入:

分析自动控制系统的数学建模过时, 根据不同专业的侧重点, 选择不同的被控对象进行数学模型的建 立;

(3) “三态” 要不忘:

整个理论体系中始终贯穿 “稳定、稳态、动态”思想。

\section{2 利用有效的教学方法, 提高学生学习兴趣}

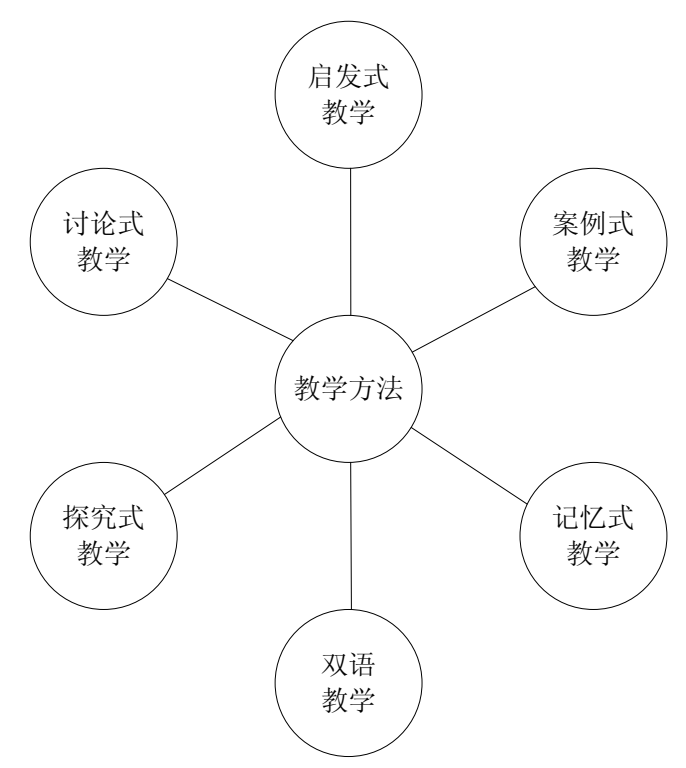

图 1 多种教学方法的汇总

兴趣是最好的老师, 调动学生的学习兴趣自然会提高教学效果。教师设定一个与学生平时生活中相关 的工程情景, 学生在教师的协助下制定解决问题的方案, 以小组交流和讨论的形式来分析和解决问题, 引 起学生的思考和探索兴趣, 联系生活实际来加深学生对理论的理解和认识, 教师与学生采取商讨式教学模 
式, 每个人都可以说出自己的想法和建议, 对积极参与讨论的学生给予一定的奖励政策, 鼓励大家多发言, 多思考, 图 1 所示为该优化方案中引入的多种教学方法。

\section{3 多种教学手段充分结合, 各显奇效}

除采用常规的 PPT 以外, MATLAB 仿真软件的应用也可以使得抽象的理论概念变得生动、通俗易懂。针 对某一具体系统, 利用 MATLAB 软件中的 SIMULINK 建模, 通过改变参数, 直观的观察对控制系统的影响, 加深对知识的印象。网络课程建设也为理论课程建设注入了新鲜血液。

\section{2 实践教学方面}

实践教学的目的是培养学生的创新精神, 启发学生思维, 激发学习兴趣, 只有建立系统的实践教学体 系, 才能真正地顺应培养工程教育人才的宗旨。以项目来带动知识点的学习, 删减基础性和验证性实验, 提高综合性和设计性实验的开出量, 增加设计研究性实验。

研究性实验可以设计为有一定难度的创新性实验, 基础好的学生可以跟随教师做一些自动控制相关的 工程项目, 不同层次的学生选择不同层次难度的实验, 满足各类学生的需要。图 2 所示的是控制理论平台 的具体内容。

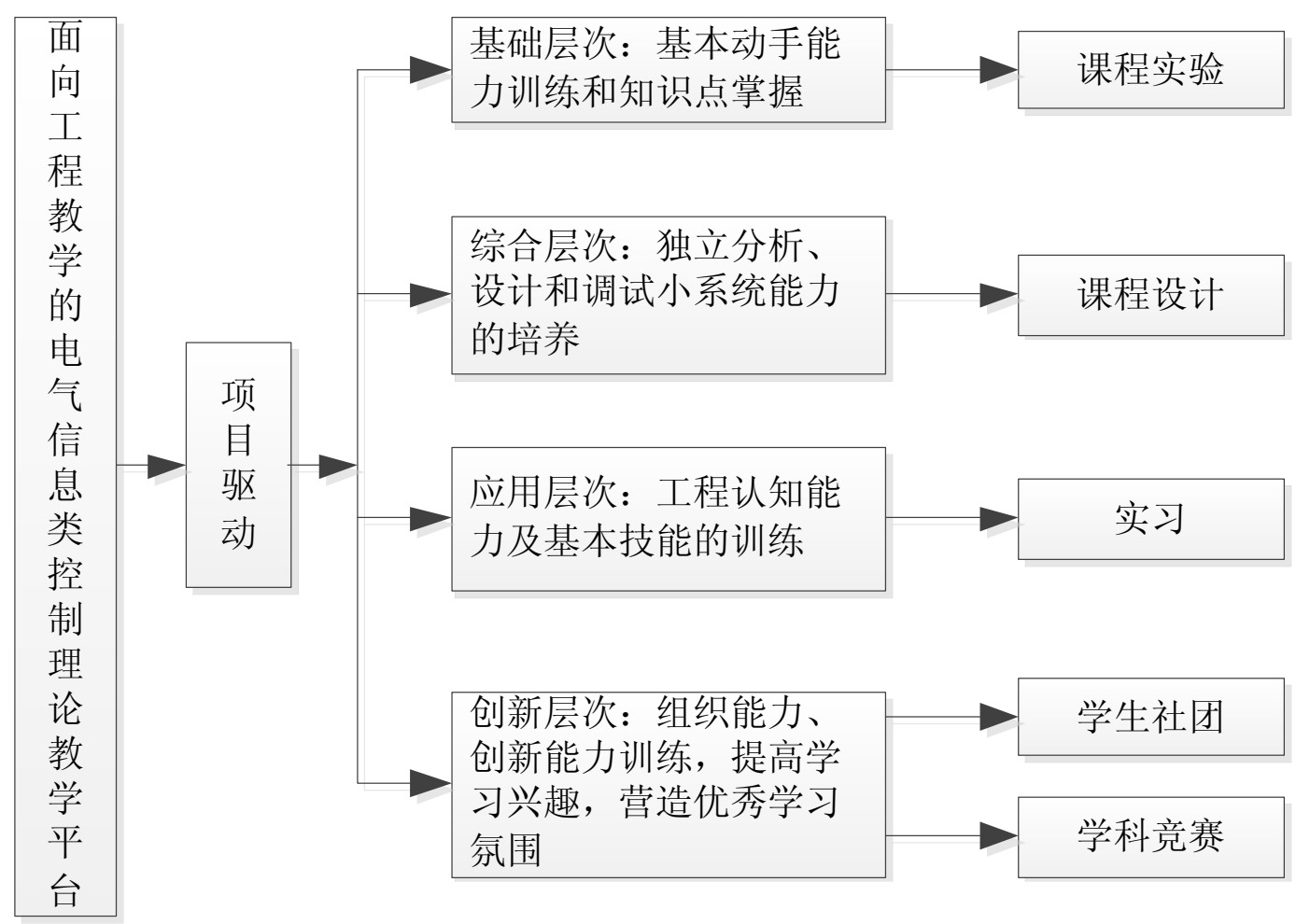

图 2 电气信息类控制理论教学平台的结构

精简优化后的理论教学内容紧贴工程实际, 使学生掌握理论知识的同时能够把书本理论嫁接到工程实 践当中去, 以点带面, 面面关联做系统。综合网络平台和先进教学手段, 融进先进前沿领域知识, 改进实 践教学环境, 突出培养学生的动手、创新力, 加强管理和考核方式, 注重考查学生的应用和创新力。

\section{3 结论}

本电气信息类教学实践平台自建成后, 在我校及各大兄弟院校进行了推广, 从学生的动手和创新能力 
及考试等多方面来看, 均好于过去的教学效果。实践出真知, 我们还应该在今后的教学中, 不断改进和优 化目前的教学体系, 为培养适应 CDI0 模式的工程类人才做出自己的努力。

\section{4 致谢}

本文的工作受到了以下项目的支持：

1）吉林省吉林市北华大学电气信息工程学院 BHDQ31;

2）吉林省教育厅科技技术研究项目（201658）;

3 ）吉林省教育厅科技技术研究项目（201656）。

\section{Acknowledgements:}

This work is supported by:

1. BHDQ31.Jilin province, Jilin, BeiHua University

2. Jilin Province Department of Education Science and Technology Plan Projects. (201658)

3. Jilin Province Department of Education Science and Technology Research Project(201656)

\section{参考文献:}

[1]马廷奇, 产学研合作与创新人才培养, 中国高等教育, 2011.6:44-46.

[2]姚聪莉, 任保平, 创新人才培养的逻辑及其大学教育转型, 中国高等教育, 2012. 7:9-12.

[3]戚业国, 大学创新人才培养体系改革的深层次思考, 中国高等教育, 2010.17:16-19.

[4]黄建钢，培养模式创新与创新人才培养，中国高等教育，2010. 9:41-42.

[5]黄德宽,创新人才培养需要制度保障, 中国高等教育, 2006. 20:17-19.

[6]段远源，张文雪,创新人才培养模式 着力培养创新人才，中国高等教育，2009. 1:25-29.

[7]刘红宁, 左铮云, 吴晓明等, 培养模式在观念贵在践行, 中国高等教育, 2010.1:25-29.

[8]姜生元, 高新, 孙丽霞等, 大学生科技创新实践基地的建设与运行, 中国高等教育, 2010.18:44-46.

[9]蒋毅坚, 地方工科院校应用型创新人才培养的研究与实践, 中国高等教育, 2010.13、14:30-32.

[10]陈初升, 蒋家平, 刘斌, 个性化长周期三结合致力于拔尖创新人才培养, 中国高等教育, 2010.21:17-19.

\section{References}

[1] Ma Ting-qi, Cooperation of Production, Teaching and Research and Cultivation of Innovative Talents , China Higher Education, 2010.9 p.41-42.

[2] Yao Cong-li, Ren Bao-ping, The Logic of Cultivating Innovative Talents and the Transformation of University Education, China Higher Education,2012.7 p.9-12

[3] Qi Ye-guo, Deep Thinking on the Reform of Innovative Talents Training System in Universities, China Higher Education, 2010.17p.16-19

[4] Huang Jian-gang, Cultivating Pattern Innovation and Cultivating Innovative Talents, China Higher Education, 2010.9p.41-42

[5] Huang De-kuan, Innovative Personnel Training Needs System Guarantee ,China, higher education, 2006.20p.17-19

[6] Duan Yuan-yuan, Zhang Wen-xue, Innovative Talents Training Mode To Cultivate Innovative Talents, China Higher Education, 2009.1p.25-29

[7] Liu Hong-ning, Zuo Zheng-yun, Wu Xiao-ming, The training model focuses on the concept of your practice, China Higher Education,2010.1p.25-29 
[8] Jiang Sheng-yuan, Gao Xin, Sun Li-xia, Construction and Operation of Science and Technology Innovation Practice Base for College Students, China Higher Education,2010.18p.44-46

[9] Jiang Yi-jian, Research and Practice on the Cultivation of Applied Innovative Talents in Local Engineering Colleges, China Higher Education,2010.13, 14p.30-32

[10] Chen Chu-sheng, Jiang Jia-ping, Liu Bin, Personalized long - term combination of three is committed to top - notch innovative talents training, Personalized long - term combination of three is committed to top - notch innovative talents training.2010.21p.17-19 